livraisons

d'Histoire

de l'Architecture

\section{Livraisons de l'histoire de l'architecture}

13 | 2007

Architectures des établissements d'enseignement supérieur

\title{
La faculté des Sciences saint-Charles À Marseille : le grand œuvre de Victor Blavette
}

The Faculty of Science at Saint-Charles : the masterpiece of Victor Blavette? Die Fakultät der Wissenschaften Saint-Charles in Marseille : das Meisterwerk Victor Blavettes?

Florence Marciano

\section{CpenEdition} Journals

Édition électronique

URL : http://journals.openedition.org/lha/412

DOI : $10.4000 /$ /ha. 412

ISSN : 1960-5994

Éditeur

Association Livraisons d'histoire de l'architecture - LHA

Édition imprimée

Date de publication : 10 juin 2007

Pagination : 89-103

ISSN : 1627-4970

Référence électronique

Florence Marciano, «La faculté des Sciences saint-Charles À Marseille : le grand œuvre de Victor Blavette », Livraisons de l'histoire de l'architecture [En ligne], 13 | 2007, mis en ligne le 10 juin 2009, consulté le 30 avril 2019. URL : http://journals.openedition.org//ha/412 ; DOI : 10.4000//ha.412

Ce document a été généré automatiquement le 30 avril 2019.

Tous droits réservés à l'Association LHA 


\section{La faculté des Sciences saint-Charles À Marseille : le grand œuvre de Victor Blavette}

The Faculty of Science at Saint-Charles : the masterpiece of Victor Blavette?

Die Fakultät der Wissenschaften Saint-Charles in Marseille : das Meisterwerk

Victor Blavettes?

Florence Marciano

1 La faculté des sciences Saint-Charles à Marseille a été construite par Victor-Auguste Blavette (1850-1934), second Grand prix en 1878 et Grand prix en 1879. Nommé architecte en chef des bâtiments civils et des palais nationaux, «sa carrière, très brillante, fut surtout une carrière administrative et professorale $»^{1}$. Il a reçu en effet peu de commandes publiques ou privées et la faculté des sciences de Marseille constitue donc le seul grand chantier d'un bâtiment neuf de sa vie d'architecte. À sa création en 1855, la faculté est installée dans des locaux appropriés par l'architecte de la Ville. L'afflux des étudiants, la nécessité de moderniser les locaux et un enseignement en perpétuelle évolution, en lien avec les impératifs de la vie économique et industrielle à Marseille avaient amené le conseil de la faculté à prévoir un ensemble de bâtiments nouveaux et adaptés à ce rôle. Mais de nombreux aléas politiques et financiers vont freiner la création d'une faculté des sciences digne de ce nom dans la deuxième ville de France. Nous allons analyser quelles ont été les conséquences de cette histoire complexe, sur le projet primé présenté par Blavette lors du concours de 1906, et la façon dont il a évolué. L'architecte at-il subi ces aléas ou bien a-t-il su en jouer pour faire réaliser son œuvre la plus importante? 


\section{La faculté des sciences de Marseille : des Capucines à Saint-Charles, une histoire administrative longue et complexe}

\section{La première faculté des sciences}

2 La « loi sur l'administration de l'instruction publique» du 14 juin 1854 institue seize circonscriptions académiques et complète l'institution universitaire en installant dans les capitales régionales les facultés manquantes, par décret du 22 août 1854 : Marseille, Lille, Poitiers, Clermont-Ferrand et Nancy en bénéficient. Cette faculté, Marseille l'appelait de ses vœux : elle a failli voir le jour en mars 1847, la Ville s'empressant alors de faire étudier par son architecte, Pascal Coste, l'appropriation de deux lieux permettant son installation, en particulier le local de la Providence, situé rue Longue-des-Capucins ${ }^{2}$. Ancien bâtiment hospitalier, Pascal Coste en propose la transformation "d'après le programme fourni par le ministère de l'instruction publique ». Fidèle à ses goûts, Pascal Coste rhabille la façade en style néo-Renaissance et lui rajoute un portique corinthien.

3 Mais ce projet n'aboutit pas et à la création de la faculté en 1854, c'est l'autre bâtiment qui est choisi, celui de la maternité située aux allées de Meilhan ; le projet d'appropriation est dû à Ferrié. Le procès-verbal de réception des travaux date du 5 mars $1858^{3}$. Ce bâtiment ainsi approprié à sa nouvelle destination va servir, bon an mal an, malgré ses défauts et le manque de place, jusqu'en 1923, date du déménagement dans la nouvelle faculté des sciences à Saint-Charles. Ce bâtiment doit abriter les installations nécessaires à l'enseignement de quatre chaires (pour un effectif de 13 étudiants inscrits en 1864) : mathématiques pures et appliquées, physique, chimie, histoire naturelle, auxquelles viennent s'ajouter en 1856 la mécanique rationnelle et appliquée et, en 1859, la géologie et la minéralogie. En 1898, la faculté dispose de treize chaires magistrales et accueille 177 étudiants; en 1907, elle compte 3210 inscrits $^{4}$.

\section{La nouvelle Faculté : des prémices à la construction}

En fait, dès la fin des années 1870 , on songe à agrandir les locaux de la faculté devenus trop exigus. La municipalité Brochier élue en janvier 1881 entame des démarches auprès du ministère dès 1882 ; l'État doit participer au projet à hauteur du tiers de la dépense, "décision alors exceptionnelle $»^{5}$. Elle vote l'installation de la faculté au boulevard de l'Observatoire (à côté du palais Longchamp), sur un terrain de $9000 \mathrm{~m}^{2}$, pour un budget de 2200000 francs. Mais malgré cette décision et la pose de la première pierre le 19 août 1883, en présence du ministre de l'instruction publique et des beaux-arts, le projet est vivement critiqué : éloignement du centre-ville, projet coûteux et difficultés financières (le préfet n'autorise que les travaux de terrassement), désaccord du professeur d'astronomie en faveur d'un agrandissement de l'observatoire qui jouxte ces terrains ${ }^{6}$. D'ailleurs, après la démission de Brochier en mai 1884, le bâtiment est remis en question alors que les travaux avaient été arrêtés dès le mois d'avril. En fait, un rapport de Louis Dieulafait, de 1885, insiste d'abord sur la nécessité de la création d'une université à Marseille en y transférant les facultés de droit et de lettres d'Aix. Le rapport conclut ensuite en six points : la faculté ne sera pas construite à Longchamp; la résiliation du contrat entre la ville et l'entrepreneur est approuvée ; la négociation doit être entamée 
avec le ministère de l'instruction publique pour l'installation d'une université complète à Marseille ; les plans de cette université doivent être mis au concours ${ }^{7}$; les bâtiments de la faculté des sciences et de médecine seront construits immédiatement; ceux des deux autres disciplines (droit et lettres) le seront ultérieurement ${ }^{2}$. Ce rapport est approuvé par délibération du conseil municipal, le 29 décembre. Les bases du futur établissement sont donc jetées : l'emplacement de la faculté des sciences ne changera plus, c'est celui des terrains de l'ancien cimetière Saint-Charles, à côté de la gare ${ }^{8}$. Il a cependant été envisagé d'autres solutions: un rapport préconise de se rapprocher de la Société immobilière marseillaise pour créer la faculté rue de la République9.

5 Il faudra encore une dizaine d'années pour que le projet soit relancé : le 18 mai 1894, le conseil municipal relance le projet de reconstruction de la faculté en lui attribuant 17000 $\mathrm{m}^{2}$ des terrains de l'ancien cimetière et en mettant en place le concours d'architecture. Pourtant, les oppositions sont tenaces: un rapport du 7 juin 1895 plaide «la perte de temps considérable et une perte d'argent absolument considérable » car il ne s'agit pas de construire une sorte de palais « où l'imagination des architectes, où l'esprit d'invention [...] consacre la gloire de son auteur et attire les regards émerveillés de la foule et des étrangers ${ }^{10}$. D'ailleurs, ce rapport préconise de s'informer des programmes et plans des facultés récemment construites, comme Lille (construite en 1887) et Nancy, et de s'en servir de type et de modèle. La présence dans les archives de plans de la faculté de Nancy (en fait, l'Institut de chimie) et celle de plans plus sommaires de la faculté de Poitiers (des locaux anciens appropriés), prouvent que la démarche a été faite. Il semble pourtant que l'organisation des bâtiments soit plutôt le reflet des questionnements sur l'organisation des universités allemandes et leur enseignement ${ }^{11}$. Le conseil de la faculté du 23 juillet 1895, sous l'égide du doyen Charve, remet la version définitive de son programme, approuvé par le ministère de l'instruction publique et des beaux-arts. Il est ouvert le 10 décembre 1895, jugé les 14 et 15 avril 1896 et le premier prix attribué à Victor Blavette. Ironie du sort, dès le 18 avril 1896, une lettre de Blavette annonce : «Je serai à Marseille jeudi ou vendredi prochain pour me mettre à votre disposition et prendre mes ordres ${ }^{12}$, alors qu'il faudra encore quinze ans pour que les travaux démarrent. En fait, les réserves émises sur le quartier de la future faculté, jugé «trop excentrique et délaissé », tout comme les éternels problèmes de financement, retardent encore la décision à prendre ${ }^{13}$.

6 En attendant, faute de place, la faculté est obligée en 1900 d'occuper, pour ses cours, l'appartement du doyen parti à la retraite. Et, en 1904, elle fait l'acquisition de l'ancien couvent des Carmélites situé rue Reinard ${ }^{14}$. Elle va réitérer ses démarches auprès de la municipalité pour acheter des terrains complémentaires ou se voir attribuer les bâtiments désormais vides du séminaire et de l'évêché (en janvier 1907). Cela lui est systématiquement refusé car la Ville maintient son grand projet de Saint-Charles.

7 En 1907, Blavette s'adresse au maire afin de savoir quelle suite il propose de « donner aux études qui ont été commandées par lettre de son prédécesseur des 7 novembre 1896 et 29 novembre 1901 pour la construction de la faculté des sciences $»^{15}$. En effet, en 1900, la Ville entame de nouvelles négociations pour construire l'Institut de chimie seulement, à condition que l'État en finance la moitié et annule sa dette de 440000 francs des versements de 1883 et 1884, mais ces négociations n'aboutissent pas.

Finalement, le vote, le 25 mars 1910, d'un emprunt de 2500000 francs permettra enfin de lancer le projet. Les plans sont approuvés le 24 janvier 1911 et les adjudications faites le 30 mars (à raison de neuf lots pour les trois instituts). L'heure est enfin à l'optimisme : «On peut espérer qu'en 1915 la faculté des sciences entrera en possession de locaux tels 
que n'en possède aucune faculté de province $»^{16}$. Mais le bâtiment des services généraux est d'emblée exclu du projet évalué lors du vote de l'emprunt, en accord avec Blavette ; sa construction est remise à plus tard. Il en va de même pour l'équipement d'un calorifère ${ }^{17}$. Les services administratifs sont alors répartis dans l'Institut de mathématiques et de physique, la bibliothèque restant dans les locaux de l'ancienne faculté18. Il faut penser aussi que la faculté des sciences doit consacrer une partie importante de son budget à la fourniture des « installations spéciales d'appareils divers ", évaluée à 500000 francs : les tables d'expérimentation dans les laboratoires disposent en effet de hottes spéciales permettant d'évacuer les émanations.

9 Les bâtiments sont encore en construction à la déclaration de guerre. Occupés par l'armée qui en a fait un hôpital militaire, deux procès-verbaux de constatation des dégâts occasionnés par l'occupation militaire, en décembre 1917 puis en juin 1919 font état d'un montant de travaux de plus de 180000 francs. Les travaux d'électricité ne sont faits qu'en 1922 et la faculté déménage pour la rentrée de 1923.

\section{La faculté des sciences de Victor Blavette: de 1896 à 1923, un projet simplifié}

\section{Le concours : un projet complet pour une faculté moderne}

10 Le programme de la faculté des sciences se partage entre quatre instituts, chimie (chimie générale, chimie industrielle et chimie agricole), physique, sciences naturelles (géologie et minéralogie, zoologie, botanique), mathématiques pures et appliquées; tous ces bâtiments abritent salles de cours et de recherche, amphithéâtre, salles de collections, bureaux des professeurs. S'y ajoute un bâtiment pour les Services généraux. Il faut prendre en compte également les locaux nécessaires au « PCN », c'est-à-dire le certificat d'études de physique, chimie et sciences naturelles obligatoire pour les étudiants en médecine.

Les trois lauréats sont dans l'ordre Victor Blavette, Bruno Pellissier et Paul Normand.

Les plans dont nous disposons datent tous de mars 1897 (ill. 1); les archives n'en ont conservé aucun, émanant du concours lui-même (à part une série de quatre photographies montées sur carton). 


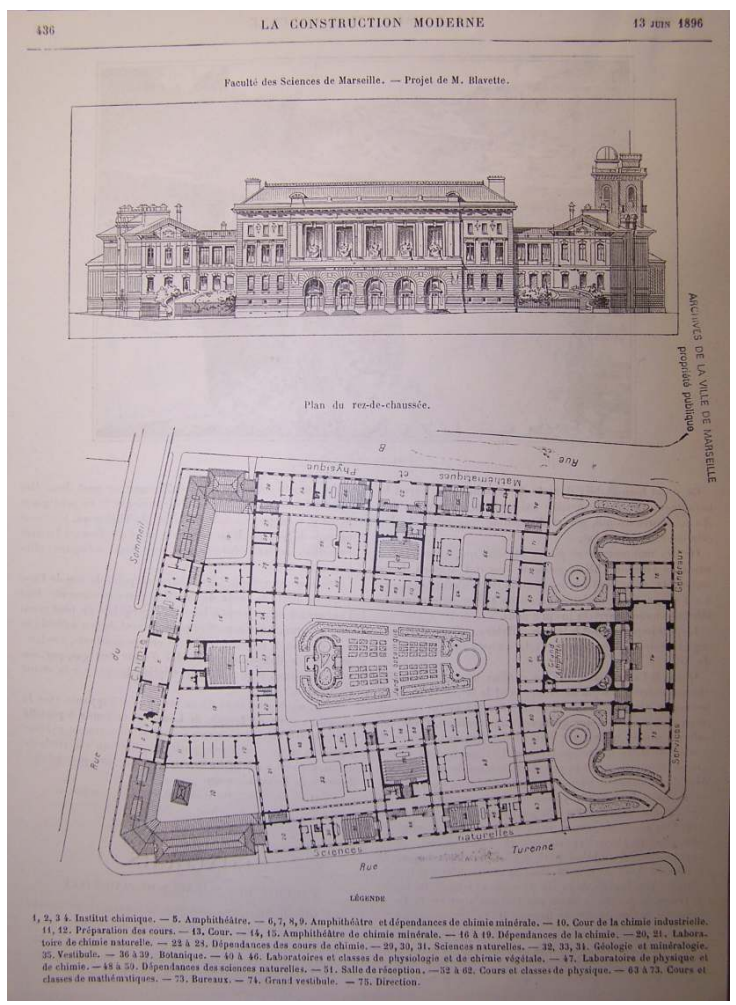

$7^{\mathrm{er}}$ prix - M. Blavette, Architecte ». Les Concours publics d'architecture, 1896, pl. 59-63. Élévation, coupe, plans

Cl. Florence Marciano

Le rapport sur les opérations du jury, dont Ernest Paugoy est le rapporteur, précise que les récompenses ont été décernées au premier tour de scrutin :

Le projet de M. Blavette se recommande tout d'abord par la rigoureuse observation de la situation de l'emplacement et des exigences du programme [...] toutes les difficultés résultant des niveaux différents de terrain, ont donc été hardiment et admirablement vaincues. La disposition d'ensemble est simple et harmonieuse; elle accuse nettement les groupes demandés. [...] La façade principale, flanquée de deux ailes, est d'une note suffisamment monumentale qui promet de gagner à l'étude et de devenir à la fois élégante et sévère. L'ensemble, avec des couvertures saillantes et en tuiles, s'inspire judicieusement des moeurs de la construction locale. [...] Son projet [...] dominait sans conteste possible le concours $^{19}$.

Le jury souligne également qu'il a été particulièrement attentif à deux points : la disposition des bâtiments sur un terrain formant un socle par rapport aux voies qui le définissent et la position des bâtiments, "parfaitement distincts [...], distribués et orientés selon les nécessités scientifiques et pouvant être desservis séparément ».

Le plan de Blavette est en effet plus immédiatement lisible que celui de Pellissier : il détache nettement le bâtiment d'accueil et répartit les trois instituts tout autour, en une composition dense où chaque ensemble de bâtiments, composé de deux ailes parallèles comprend deux cours intérieures séparées par l'amphithéâtre. Il regroupe mathématiques et physiques dans la partie droite, les sciences naturelles étant en face (l'institut est réparti en trois disciplines : géologie et minéralogie, zoologie, et botanique) et l'Institut de chimie fermant le trapèze. En contrebas, deux ailes d'un seul étage 
l'encadrent, pratiques car « de plain-pied avec les rues latérales ». Cette même disposition se trouve chez Pellissier; elle est conforme aux indications du programme : «La Ville de Marseille compte réaliser, avec l'intervention de l'État, la construction de cet ensemble en procédant successivement. C'est l'Institut de chimie qui serait exécuté le premier. Au fur et à mesure des ressources, les autres parties du projet seront en voie d'exécution ${ }^{20}$. » Il doit donc être indépendant des autres. Blavette utilise intelligemment la grande cour centrale pour y installer le jardin botanique, une serre et un vivier. Tous deux prévoient de passer d'un bâtiment à l'autre par des portiques, qui complètent un dispositif de circulation "qui est l'une des qualités primordiales du plan »; on le félicite aussi pour ses «bâtiments bien éclairés, aérés et ventilés». Pellissier, en revanche, dessine des bâtiments en simple profondeur, ce qui l'oblige à disposer une quatrième aile derrière le bâtiment des Services généraux.

Blavette prévoit deux tours côté Institut de physique, l'une pour la météorologie et l'autre, symétrique, pour l'astronomie, suscitant ce commentaire: "une connaissance accomplie des besoins des services et le soin apporté à l'étude jusques dans ses moindres détails (il n’a pas non plus négligé de prévoir un chenil et une salle de vivisection!). Curieusement, c'est la deuxième tour que Pellissier dessine également, alors que rien dans le programme n'a trait à l'astronomie.

17 Les élévations jouent à la fois sur l'unité et la diversité : symétrie de chaque façade, ouvertures régulièrement disposées, ligne générale continue des niveaux des façades grâce à des cours anglaises, mais comme chaque aile et chaque institut ont leur propre programme, on différencie les façades. Il refuse la monumentalité pour les façades sur rue, contrairement à Pellissier. Pour les façades intérieures, l'Institut de chimie reçoit un avant-corps central avec un double portique de quatre colonnes sur deux étages surmonté d'une horloge.

Quant à la façade principale, il respecte les souhaits formulés par la Ville d'un bâtiment simple: un avant-corps sur deux étages, percé de cinq grandes portes donnant sur le vestibule d'honneur. À l'étage, les grandes fenêtres encadrées de pilastres permettent d'éclairer la salle de lecture de la bibliothèque; peu de décor : deux petits frontons et six trophées.

\section{Les projets de 1902 et 1910 : vers la simplification}

19 La deuxième série de plans de Blavette, pour le projet de la faculté, date de 1902. En 1900, la Ville propose au ministère de construire immédiatement l'Institut de chimie, ce qui oblige Blavette à envoyer en avril 1902 un dossier complémentaire de 17 feuilles de dessins et 5 devis. Les distributions sont les mêmes, mais on remarque déjà quelques simplifications: par exemple, l'abandon de deux petits bâtiments accolés à celui des amphithéâtres (salles des machines, atelier) au bénéfice de deux escaliers en fer à cheval menant directement au premier étage. La tour de l'astronomie n'existe plus; seule subsiste, au centre de l'élévation sur la rue «B» (l'actuelle rue Desplaces) la tour de la météorologie (ill. 2 et 3). Les articulations ont changé entre les instituts, avec des escaliers dans les angles. 
III. 2 : « Institut de chimie. Élévation sur le jardin botanique»

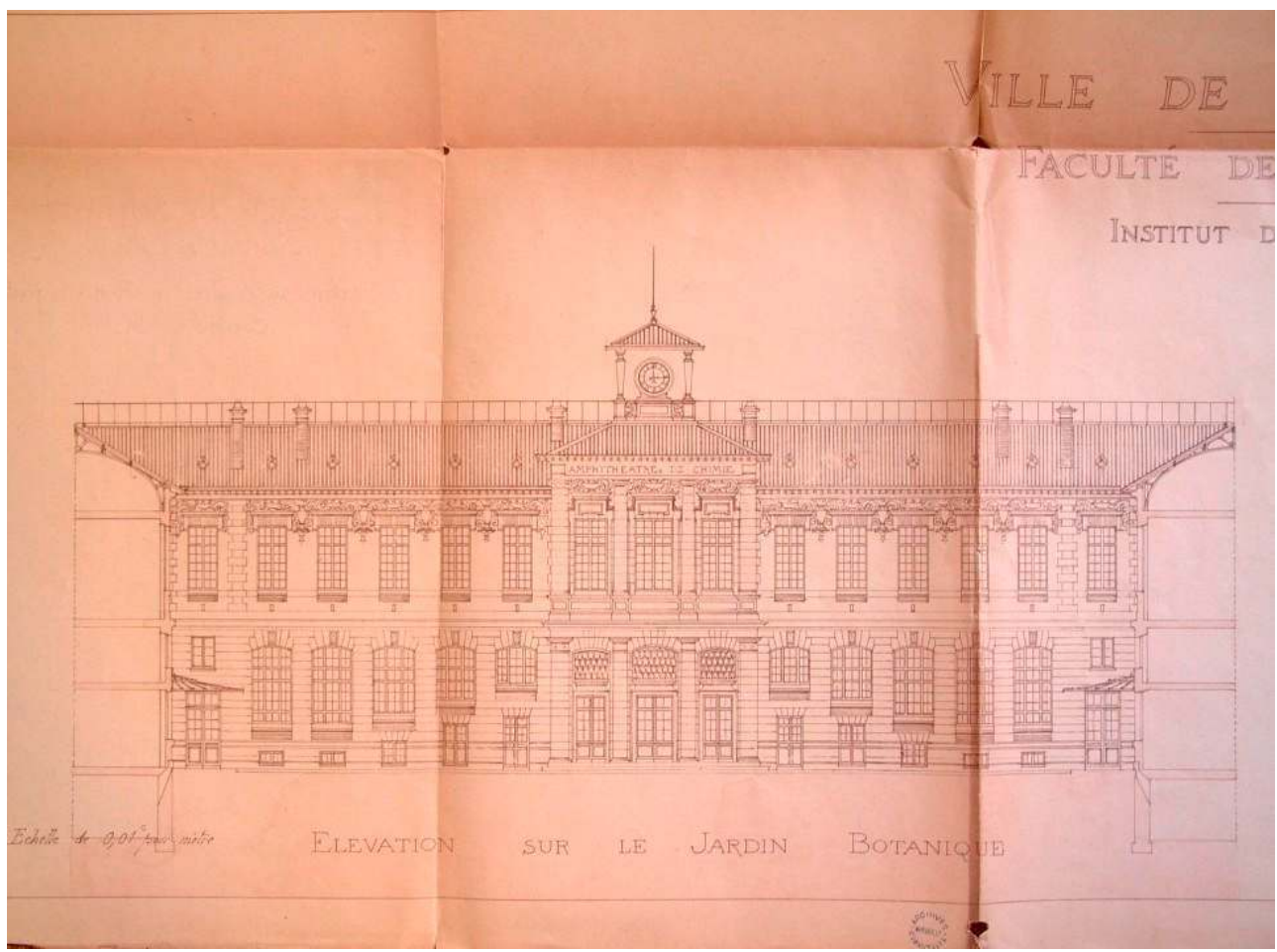

Dessin de Blavette, 10 mars 1897, Arch. mun. Marseille, 94 M 2 bis

$\mathrm{Cl}$. Florence Marciano

III. 3 : « Faculté des sciences. Élévation sur la rue B »

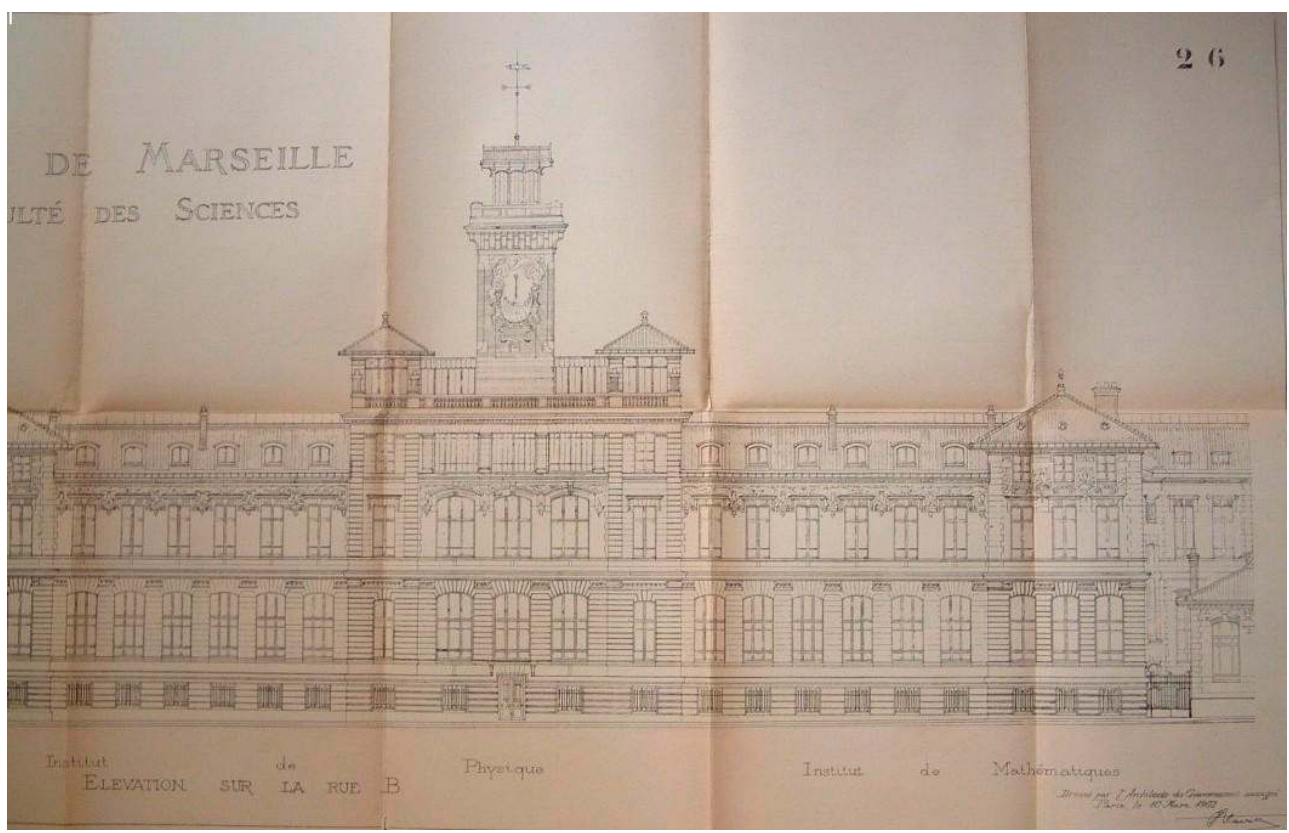

Dessin de Blavette, 10 mars 1902, Arch. mun. Marseille, 94 M 6

$\mathrm{Cl}$. Florence Marciano

En mars 1910, sur la demande du ministère de l'Instruction publique, l'architecte doit lui soumettre des modifications de ses plans, «comme conséquence de l'acquisition [...] de 
locaux où ont été installés des services dont le transfert à l'ancien cimetière Saint-Charles était prévu au programme de $1895 \aleph^{21}$. Paradoxalement, les initiatives prises par Charve pour pallier les atermoiements de la Ville, malgré ses promesses de construction d'une grande faculté moderne, aboutissent à un programme amoindri. Parallèlement, dès 1909, le conseil de la faculté avait demandé à Blavette de revoir également l'organisation des bâtiments avec des instituts séparés, ce qu'il accepte de faire.

Dès lors, la série des plans de novembre 1911 présente un visage très différent (ill. 4 et 5).

\section{4 : « Faculté des sciences. Plan général - $1^{\text {er }}$ étage »}

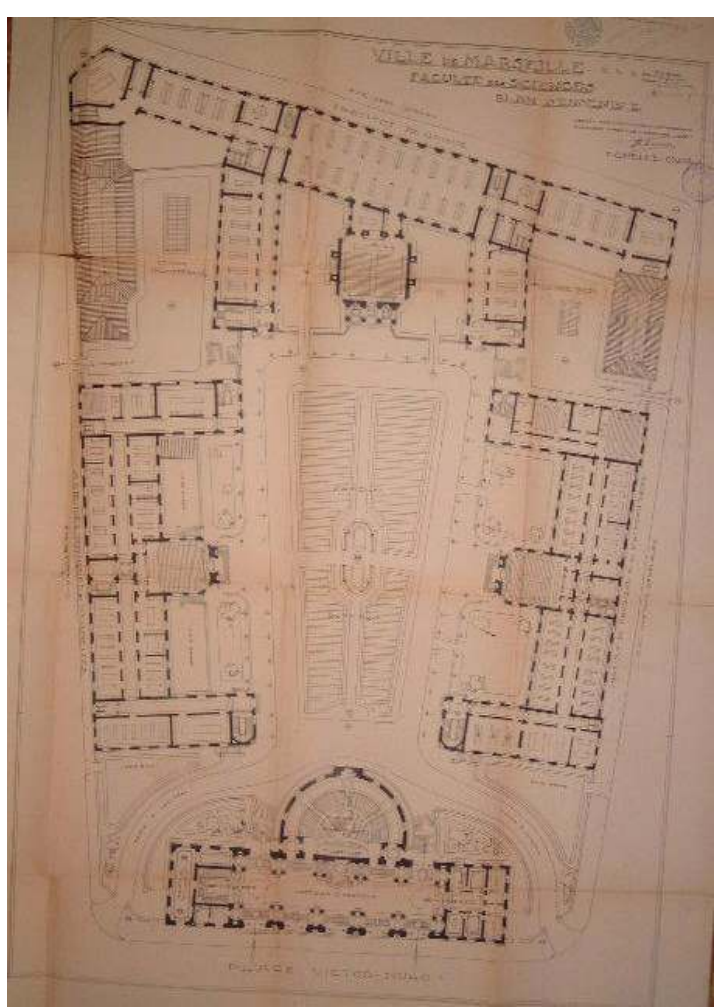

Dessin de Blavette, 5 novembre 1910. Arch. mun. Marseille, 94 M 3

$\mathrm{Cl}$. Florence Marciano 
III. 5 : « Faculté des sciences. Élévations longitudinale et transversale sur cour »

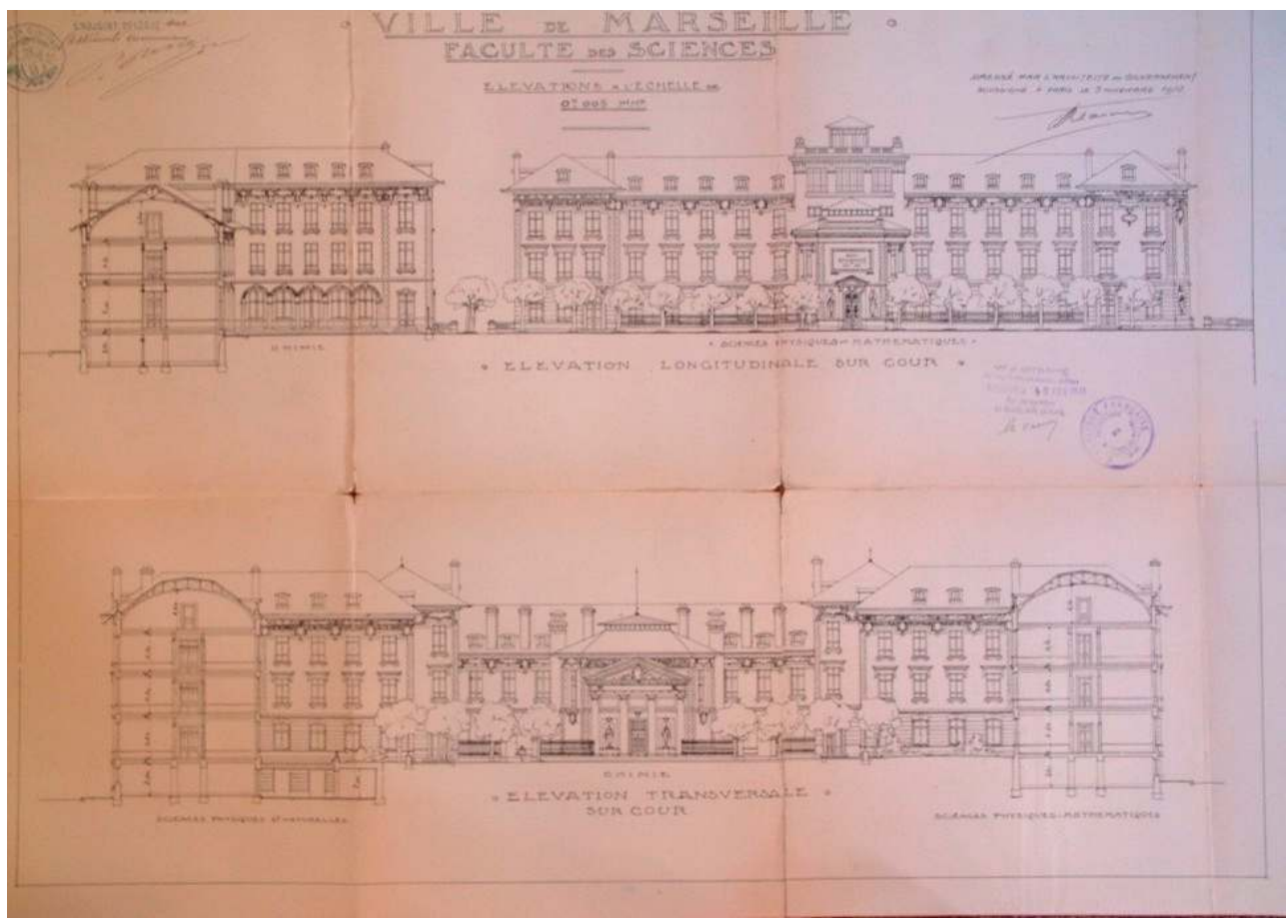

Dessin de Blavette, 5 novembre 1910. Arch. mun. Marseille, 94 M 3

$\mathrm{Cl}$. Florence Marciano

La première chose à remarquer est la présence du bâtiment des Services généraux, que Blavette a maintenu pour l'équilibre de son plan. La différence majeure est la surface des bâtiments: dans un plan d'ensemble semblable, Blavette répond aux demandes de révision qui lui ont été faites en séparant nettement les trois instituts, ce qui l'oblige à diminuer la longueur des bâtiments en dédoublant la profondeur ; on passe, de deux ailes parallèles, à un plan en E. Les lignes générales des élévations sont identiques. Travées régulières, rez-de-chaussée à bossages séparés par un gros cordon, chaînages d'angle, baies en arc segmentaire et rectangulaires, grands toits de tuiles. Le changement principal se voit dans la réduction systématique du nombre de travées, donc dans l'ampleur des bâtiments ${ }^{22}$. Cela oblige l'architecte à changer l'organisation de la façade rue de Turenne: plus de pavillons d'angle, mais une travée centrale marquée par un fronton. On note l'apparition des nombreuses cheminées sur le toit de l'Institut de chimie, qui témoignent des aménagements modernes d'évacuation des émanations au-dessus des tables d'expérimentation.

Par ailleurs, il est troublant de constater de multiples changements de détail dans l'élévation des différents instituts. L'exemple le plus frappant est celui des avant-corps centraux : les trois sont identiques, finalement construits sur le modèle de celui de la chimie ; ils ne sont pas couverts d'un dôme, mais d'un toit de tuiles à quatre pentes. Le pan coupé sur la rue de Turenne est percé d'une arcade de baies en plein-cintre, séparées par une demi-colonne engagée. 


\section{Les transformations successives du bâtiment des services généraux} propositions pour diminuer le prix de la construction. Au contraire, les changements opérés montrent un enrichissement de l'élévation, premier étage portant des pilastres (jumelés ici) et petits frontons dans les travées extérieures. Il transforme la ligne de toit en rajoutant un rang de balustres scandé par quatre paires de pots à feu avec, en amortissement, deux petits obélisques. Les toits sont percés de grandes lucarnes. Les façades des ailes, dans la perspective, sont également différentes (ill. 6).

\section{6 : « Faculté des sciences. Services généraux»}

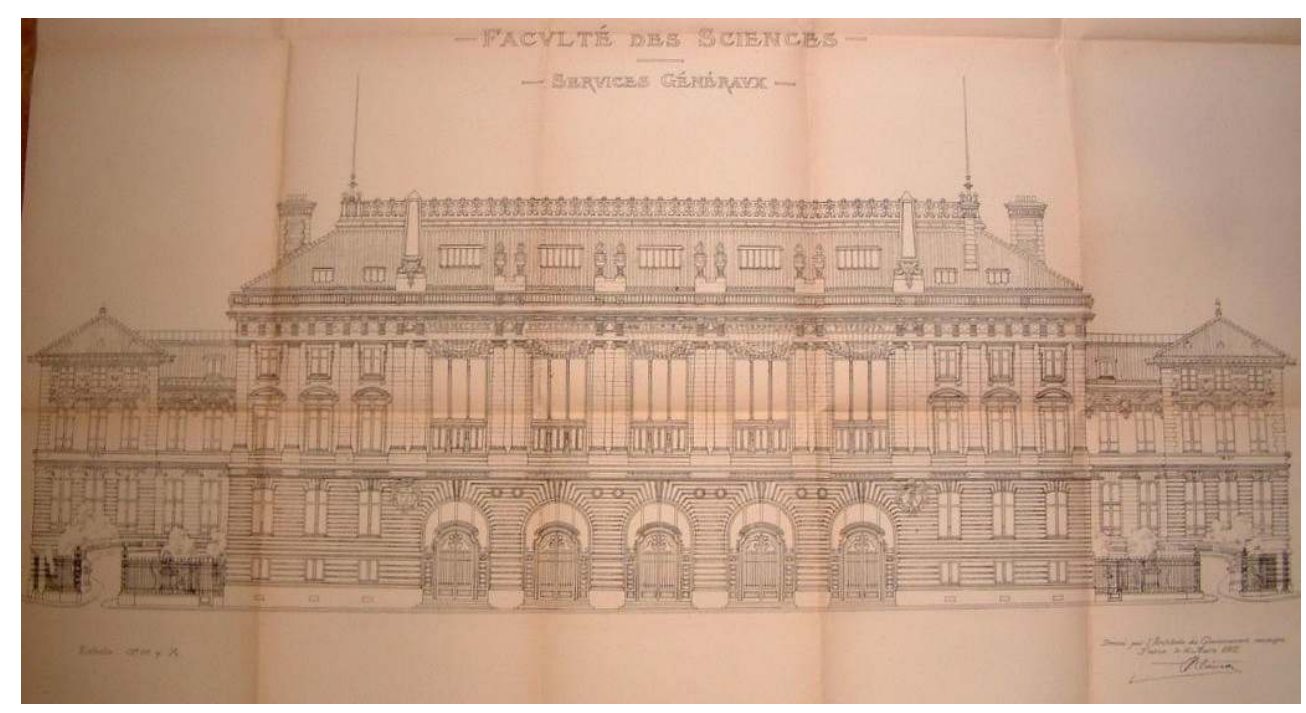

Élévation de la façade, dessin de Blavette, 10 mars 1902, Arch. mun. Marseille, 94 M

Cl. Florence Marciano.

Le devis qui accompagne les plans indique la part consacrée à la sculpture : «deux écussons avec armes de la Ville et initiales, grand cartouche couronnant les grandes baies au premier étage, sculpture sur les pylônes, huit vases [...], dans les cours : pilastres en bas des rampes, quatre lions, staffs, plafonds et murs de l'amphithéâtre ${ }^{23}$.

Sur la série des plans de 1911, les services généraux sont intégrés, malgré le consensus général pour leur abandon. Blavette en a simplifié le plan en éliminant l'escalier monumental qui menait à l'amphithéâtre et le salon de réception ; totalement détaché du reste de la composition, les galeries extérieures de circulation ne sont plus nécessaires. En 1912, il envoie même une série de plans intitulée " projet B », plus simple, pour tenter de convaincre la Ville, En 1920 encore, Blavette lui fait parvenir de nouveaux plans, «[s]e conformant à ses instructions $»^{24}$ pour passer d'un bâtiment monumental qui coûtait 2 400000 francs avant-guerre à un édifice plus simple estimé à 883189 francs $^{25}$. Il renoue pourtant avec la monumentalité, en particulier pour la façade sur jardin (ill. 7). 
III. 7 : « Bâtiment des services généraux. Élévation sur le jardin botanique »

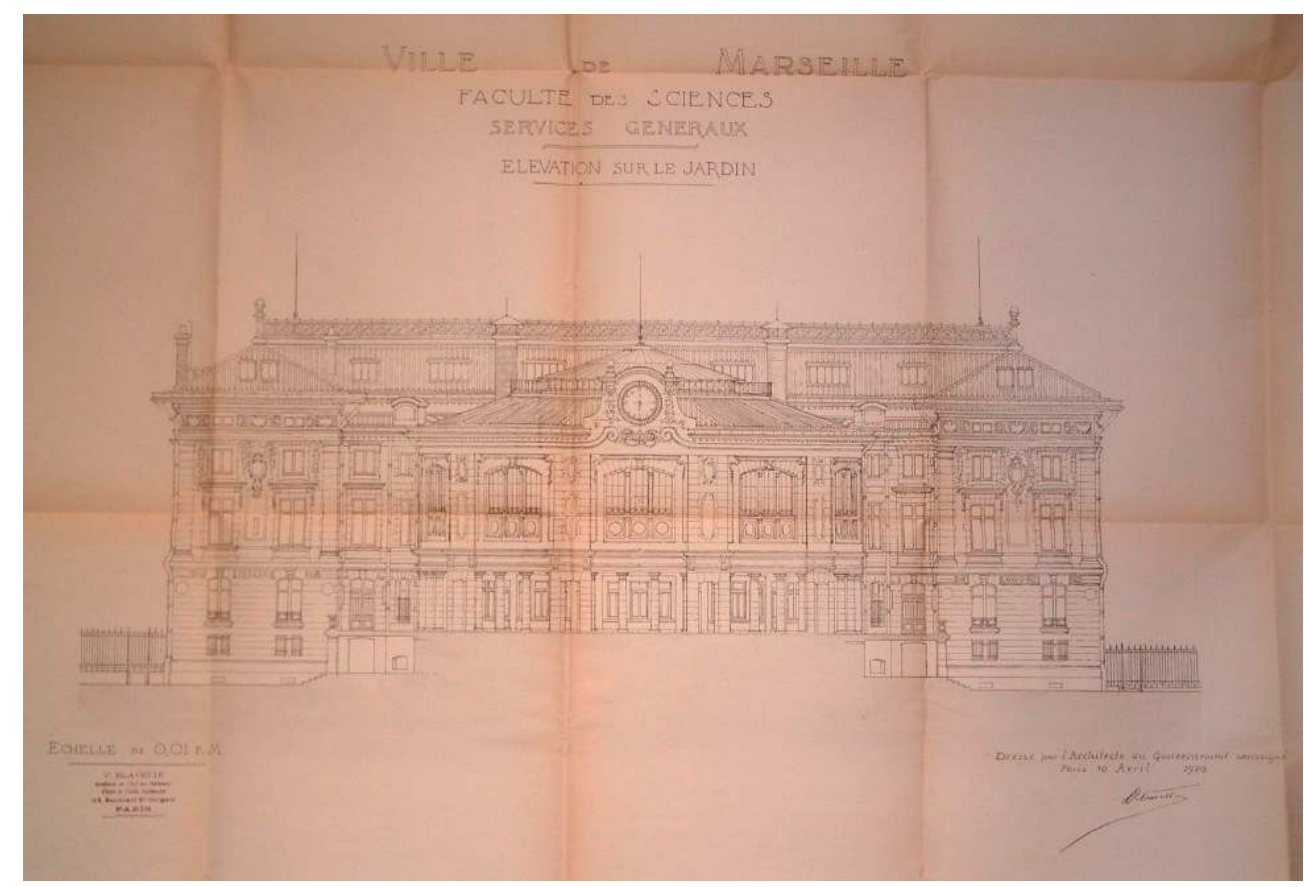

Dessins de Blavette du 10 avril 1920. Arch. mun. Marseille, 94 M 16

$\mathrm{Cl}$. Florence Marciano

\section{Le décor : projets et réalisation}

L'animation des façades se fait par l'important décor de sgraffites. Elles en sont toutes ornées: longue frise sous le toit, ponctuée de motifs ornementaux entre les aisseliers du toit et, sur chaque façade, de grands médaillons (ill. 8). 


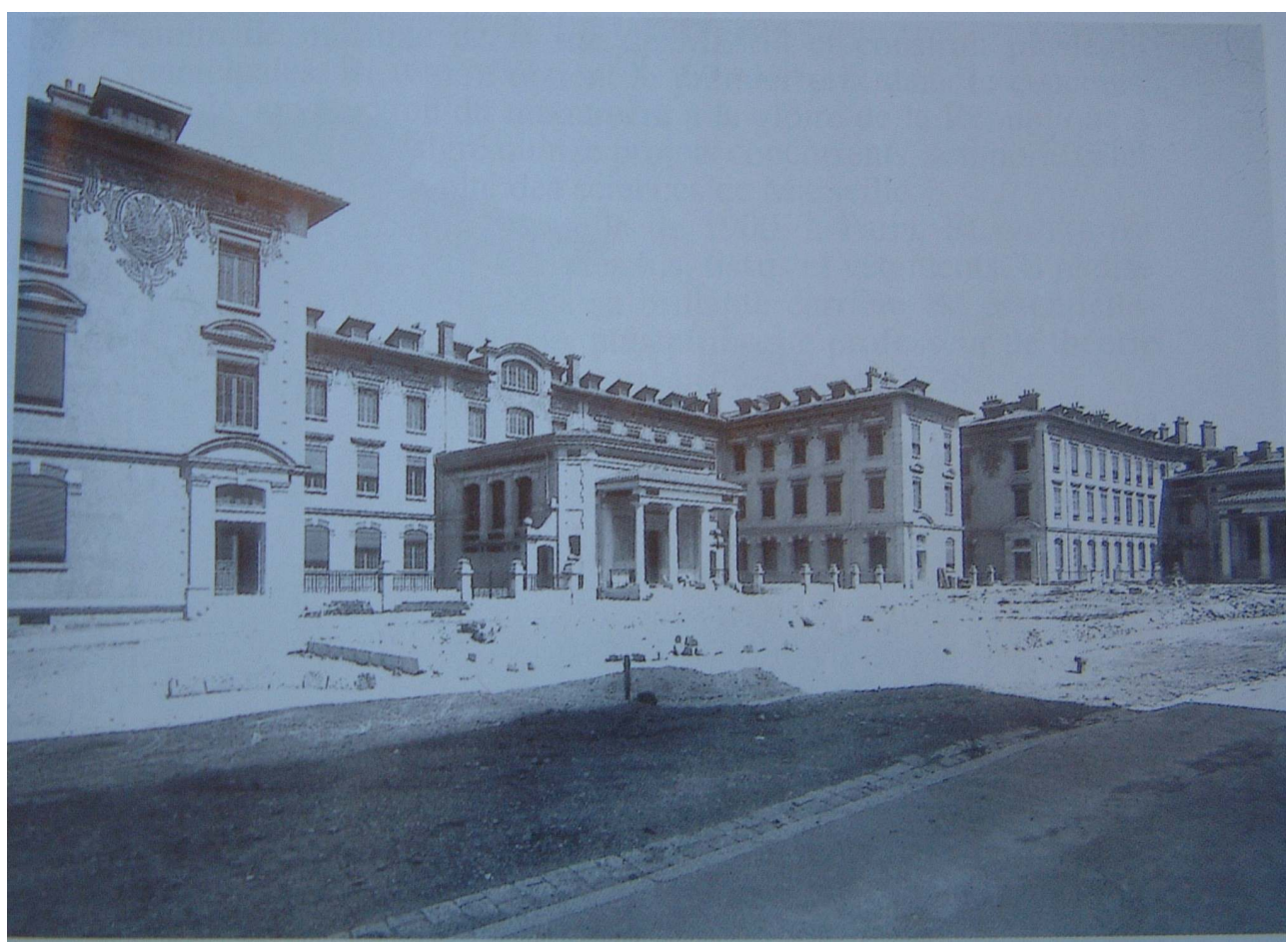

Décor de médaillon en sgraffite sur le bâtiment des sciences naturelles

$\mathrm{Cl}$. Florence Marciano

de décor est certainemer dans un environnement méridional. Le jury a apprécié en effet «les couvertures saillantes et en tuiles [qui s'inspirent] judicieusement des mœurs de la construction locale [...], une cuvre architecturale personnelle aussi originale qu'intéressante, bien appropriée au climat du Midi ». En réalité, la technique du sgraffite et le toit débordant, s'ils sont très répandus dans la région de Nice, si proche géographiquement et culturellement de l'Italie, sont très peu présents à Marseille, que ce soit dans l'architecture publique ou privée. Certes, ils commencent à se diffuser en cette fin de siècle, mais il s'agit plus d'un effet de mode que de la continuation d'une tradition. Par ailleurs, le décor se fait par l'utilisation de la brique, dans les jeux de disposition (claveaux des arcs des fenêtres, frises de dents d'engrenage).

Le décor, exécuté par Théodore-Henri Riebel (1880-1942) sur des dessins de Blavette, permet de signaler au public la fonction de chaque bâtiment : les motifs et les médaillons représentent divers instruments (un ballon de distillation pour la chimie), d'autres plus symboliques (une proue de navire pour la botanique). Ils sont traités de façon stylisée.

31 En ce qui concerne le décor intérieur, une fois la décision prise de ne pas construire « l'élément de prestige » que constituent les services généraux, il est aussi décidé en 1913 au sein du conseil de la faculté et après consultation de Blavette « qu'il n'y a pas lieu de laisser exécuter, dans les trois amphithéâtres une décoration intérieure au moyen de peintures à la fresque, comportant des figures ${ }^{26}$. Curieusement, c'est le conseil municipal qui lance les commandes de grandes fresques décoratives, sur proposition d'Alfred Lombard; celui-ci se charge du décor de l'amphithéâtre de sciences naturelles, Pierre Girieud, de celui de mathématiques et de physique, et Jean-Paul Laffitte, de celui de 
chimie $^{27}$. Les échafaudages sont mis en place au début de l'été 1914. Á leur démobilisation, les deux premiers (Laffitte est mort) s'enquièrent de leur commande; ils s'entendent répondre qu'elle est devenue «nulle du fait de la non-continuation des travaux ». Et pour cause ! La municipalité s'abrite ainsi derrière un prétendu manquement des deux artistes pour ne pas avoir à financer des travaux dans lesquels elle s'était imprudemment engagée.

La faculté des sciences installée en 1922 est issue d'un projet conçu presque trente ans auparavant. De la grande faculté des sciences, moderne, exemplaire, imaginée en 1896, Blavette a su conserver l'esprit. Le plan initial a pu subir les avatars successifs sans être vidé de son sens. Á la fin de la guerre, les visiteurs reconnaissent d'ailleurs qu' «aucune faculté des sciences française, sauf la Sorbonne, ne dispose de bâtiments aussi étendus ni mieux appropriés ». Aujourd'hui, la faculté des sciences témoigne un peu moins du talent de son architecte: la réhabilitation récente a achevé de la vider de sa substance. L'ensemble des bâtiments a subi de profondes transformations: structure intérieure bouleversée, escaliers en béton, changement de toutes les huisseries intérieures et extérieures, et des planchers. Sans compter la réfection des enduits extérieurs qui a fait disparaître le décor de sgraffites ${ }^{28}$. Seuls quelques décors ont été préservés à titre de témoignage, dans l'amphithéâtre de sciences naturelles en particulier. Du programme très complet de Blavette ne reste pratiquement qu'une coquille vide, mais l'espace de la cour intérieure et les trois pavillons démontrent encore son talent. La faculté des sciences fait pendant à l'ensemble occupant l'autre versant du plateau Saint-Charles : l'escalier de la gare de Sénès et Arnal, lui aussi un édifice «fidèle à la tradition académique par le décor architectural et le programme iconographique »; il est construit sur concours, en 1911, et inauguré en 1928. Curieusement, Marseille hérite tardivement de deux témoignages de l'architecture éclectique, avec ces bâtiments achevés au moment où apparaît le mouvement moderne.

\section{NOTES}

1. Albert Louvet, « Victor Blavette (1850-1933) », L’Architecture, 15 mars 1934, p. 33-34. Il a été architecte en chef des Palais du Louvre et des Tuileries, chargé de travaux au Conservatoire de Musique et au Muséum d'histoire naturelle; il a été professeur de théorie à l'École des beaux-arts de 1908 à 1927.

2. Arch. mun. Marseille, 93 M 1, Faculté des sciences, Capucines, 1847-1875, « Devis et détail estimatif de la construction d'une Faculté des sciences à Marseille ", $1^{\text {er }}$ février 1848.

3. Ibid., procès-verbal de réception des travaux, 5 mars 1857, signé Ferrié et Juran fils, adjudicataire des travaux. Les travaux de transformation du bâtiment ont coûté 107412 francs (construction neuve pour l'agrandissement du local, restauration des vieilles façades et ouvrages complémentaires, mur de clôture et ouvrages divers, appropriation de la maison occupée).

4. Paul Masson (sous la dir. de), Les Bouches-du-Rhône. Encyclopédie départementale, deuxième partie : Le bilan du XIXe siècle, t.VI : La vie intellectuelle, p. 104-105 : c'est-à-dire 13 
professeurs titulaires, 2 professeurs-adjoints, 1 maitre de conférences, 3 chargés de conférences, 5 chefs de travaux pratiques.

5. Henri Tachoire, «La création de la première faculté des Sciences aux allées de Meilhan », Marseille. 2600 ans de découvertes scientifiques, t. II : Vers la création de la faculté des Sciences, dir. G. Aillaud, Y. Georgelin, H. Tachoire, publications de l'Université de Provence, Aix-en-Provence, 2002, p. 172. La convention entre l'État et la Ville est signée le 8 janvier 1883.

6. Aucun plan de Paugoy ne nous est parvenu, mais seulement un plan-masse très sommaire.

7. Le projet lui-même, dont l'auteur est l'architecte de la Ville, Ernest Paugoy, avait été qualifié de sommaire et d'onéreux ; parallèlement au rapport de Dieulafait, la Ville a commandé à trois architectes, Sixte Rey, Joseph Huot et Mirande, un rapport sur l'estimation du devis (mais sans critique sur le projet lui-même); rendu le 25 février 1885, il estime les travaux à 412 241, 24 francs de plus. Ce problème explique sans doute la conviction de devoir ouvrir un concours plutôt que de confier la nouvelle construction à l'architecte municipal.

8. Le cimetière occupait une surface de neuf hectares; devenu trop petit mais trop proche de la ville pour être agrandi, depuis l'installation de la gare ; il est transféré à Saint-Pierre petit à petit, à partir de 1863.

9. Arch. mun. Marseille 94, M 2 bis, «Projet relatif à la construction d'une faculté des sciences nouvelle dans la rue de la République sur un vaste terrain de la Société immobilière peu après la place centrale». Ce rapport, ni signé, ni daté, argumente sur la centralité de la rue de la République (créée entre 1864 entre le Vieux-Port et les ports nouveaux de la Joliette) et sur l'intérêt de la Société Immobilière qui cherche à y attirer « un grand nombre d'établissements publics ». Il n'a y pas trace dans les délibérations du conseil municipal que ce rapport ait été pris en compte.

10. Arch. mun. Marseille, $94 \mathrm{M} 2$ bis, Rapport relatif à la suppression du concours, 7 juin 1895.

11. Henri Tachoire, "Léon Charve et la nouvelle faculté des sciences», op.cit., p. 201. Les références du conseil de la faculté sont A. Wurtz, Les Hautes Études pratiques dans les Universités Allemandes, Imprimerie impériale, Paris, 1870 et E. Lavisse, La fondation de l'Université de Berlin à propos de la réforme de l'enseignement supérieur en France, avec une note sur l'Université allemande de Strasbourg, Hachette, Paris, 1882.

12. Arch. mun. Marseille 94, M 2, Nouvelle faculté des sciences, lettre de Victor Blavette au maire, 18 avril 1896.

13. Marcel Roncayolo, «La croissance urbaine", Marseille au XIXe siècle. Rêves et triomphe, catalogue d'exposition, Musées de Marseille - Réunion des Musées nationaux, 1991, p. 21-41. La Ville de Marseille est alors très endettée par tous les grands travaux du Second Empire: bâtiments publics (Bourse, palais des Arts, palais Longchamp, etc.), infrastructure (canal de Marseille, nouveaux ports de la Joliette), urbanisme (Canebière, rue Impériale), situation aggravée par la crise économique de 1870-1880. Les décennies suivantes sont consacrées par la municipalité «au tout-à-l'égout, aux transports en commun, à la diffusion de l'enseignement primaire, au respect de l'hygiène publique » (p. 40).

14. Henri Tachoire, «Léon Charve et la nouvelle Faculté des Sciences », op. cit., p. 196. La faculté, grâce à l'argent d'une rente, d'un prêt et d'une subvention du conseil général et de la Ville, a pu acheter les bâtiments et un terrain attenant. On y installe un laboratoire de chimie et celui de botanique agricole.

15. Arch. mun. Marseille, $94 \mathrm{M} 2$ bis, lettre de Victor Blavette au maire de Marseille, 22 octobre 1907.

16. Les Bouches-du-Rhône. Encyclopédie départementale, op. cit., p. 69.

17. Arch. mun. Marseille, 94 M 12 bis, Bulletin municipal officiel, délibération du conseil municipal du 25 mars 1910. 
18. En fait, on peut considérer que le programme a été complété avec la construction de la bibliothèque de Fernand Pouillon en 1958. Dans le même temps, le hall des essais de l'Institut de chimie situé rue de Turenne, a subi une transformation totale : sur ses fondations est reconstruit un bâtiment en béton de quatre étages (1956).

19. Arch. mun. Marseille, $94 \mathrm{M} 2$ bis, Concours pour l'édification d'une Faculté des Sciences à Marseille. Rapport sur les opérations du jury.

20. L'Institut de chimie constitue le cœur de la faculté. Son importance est souvent soulignée dans les rapports, en particulier à cause de ses relations avec l'industrie marseillaise : Léon Charve le dit dans une lettre au maire de Marseille du 29 septembre 1906 : «L'exposition coloniale [de 1906] a montré avec éclat l'importance de l'industrie des corps gras à Marseille [...] la chaire de chimie industrielle a déjà secondé les efforts de nos savonniers qui viennent s'aider de ses conseils dont l'efficacité est malheureusement entravée par l'insuffisance de nos laboratoires ». Il s'agit de "fournir des techniciens habiles et instruits à toutes les usines de Marseille » (rapport de l'Université, 1900).

21. Arch. mun. Marseille, $94 \mathrm{M} 2$ bis, Bulletin municipal officiel.

22. L'absence de plans d'exécution et procès-verbaux de réception des travaux ne permet pas de suivre le chantier de la faculté ni de savoir finalement quel a été le budget consacré à sa construction, étant donné sa simplification.

23. Arch. mun. Marseille, 94 M 3, Devis du 10 mars 1902 pour le pavillon central des services généraux.

24. Arch. mun. Marseille, 94 M 16, lettre de Victor Blavette au maire de Marseille, 5 mai 1920. Cette phrase est soulignée en rouge et accompagnée d'un «?»; dans la marge, il est écrit: " prière de chercher qui a donné des instructions en ce sens à M. Blavette ».

25. Arch. mun. Marseille 94, M 16, faculté des sciences, bâtiment des services généraux, 1920-1923. Un rapport de la commission des bâtiments communaux et des travaux d'architecture précise que les «prix des travaux sont cinq fois plus élevés qu'avantguerre en moyenne ».

26. Henri Tachoire, "Léon Charve et la nouvelle Faculté des Sciences", op.cit., p. 204. Les membres du Conseil précisent : "Conscients du gros effort que la Ville veut bien faire en notre faveur [...], nous sommes opposés en principe à toute dépense somptuaire ».

27. Deux esquisses sont conservées dans le fonds Lombard à la bibliothèque Jacques Doucet.

28. Ils ont toutefois fait l'objet d'un relevé systématique et il a été question de les redessiner au trait, mais le coût du chantier a fait reculer l'administration.

\section{RÉSUMÉS}

La faculté des sciences Saint-Charles à Marseille, créée en 1854, a besoin vingt ans plus tard d'un bâtiment adapté à ses besoins et au nombre croissant d'étudiants. Après un premier projet voté en 1883, commencé puis abandonné, la Ville prend la décision d'ouvrir un concours national en 1895 , dont le premier prix est attribué à Victor Blavette, architecte du gouvernement. C'est la seule grande commande publique qu'il ait reçue. Mais les problèmes administratifs et surtout financiers retardent sa construction. Enfin mise en chantier en 1911, la construction est interrompue par la guerre et la faculté n'emménage définitivement qu'en 1922. L'article propose 
d'analyser en quoi la durée très longue d'exécution du projet a obligé l'architecte à revoir constamment ses plans, dont nous connaissons trois versions, 1897, 1902 et 1910. Chaque livraison montre que l'architecte les a simplifiés, soit de sa propre initiative, soit à la demande des commanditaires. L'architecte a été obligé de livrer finalement une version amoindrie du bâtiment proposé lors du concours (en particulier en éliminant le bâtiment central des Services généraux). Il a toutefois réussi à en conserver les qualités, pour créer une faculté des sciences remarquée à l'époque pour son ambition et son modernisme.

Founded in 1854, the Faculty of Science at Saint-Charles in Marseilles is in need of a building more appropriate to the increasing number of students twenty years later. After a first design voted in 1883 was started but given up, the local authority makes the decision to start a national competition in 1895, and Victor Blavette, as an architect of the governement, is awarded the first prize. This is but the only significant public commission he has ever had. But the construction is postponed by administrative and financial difficulties. As it finally gets started in 1911, the construction gets broken off by the coming of war and the Faculty does not settle in until 1922 . This article aims at analyzing to what extent the long-running project makes the architect review his designs again and again, whose three versions - ones of 1897, 1902 and 1910 - are known. Each proposition points out that the designs were simplified by the architect, either he did it on his own initiative, either he was asked to by the local authority. The architect finally had to deliver a lesser version of the planned building (particularly by cutting out the central premises of the services). Nevertheless he successfully conserves its qualities so as to design a faculty of science whose ambition and modernism do not remain unnoticed at the time.

Die 1854 gegründete Marseiller Fakultät der Wissenschaften Saint-Charles musste schon nach zwanzig Jahren mit einem neuen, der wachsenden Anzahl der Studenten angemessenen Gebäude ausgestattet werden. Das erste Projekt, 1883 genehmigt, wurde kaum angefangen aufgegeben, so dass die Stadt Marseille 1895 beschloss, einen nationalen Wettbewerb zu veranstalten, den der Staatsarchitekt Victor Blavette als erster Preisträger gewann.. Es wurde der einzige öffentliche Auftrag seiner Karriere. Aus Gründen von Verwaltungs- und Geldproblemen musste der Baubeginn bis 1911 immer wieder verschoben werden. Der 1. Weltkrieg unterbrach wiederum den Fortgang der Bautätigkeiten, so dass erst 1922 die Fakultät in ihr neues Gebäude einziehen konnte. Der Artikel untersucht, inwiefern die langwierige Projektausführung den Architekten zu ständigen Änderungen seiner Baupläne zwang. Je nach den drei bekannten Entwürfen 1897, 1902 und 1907 erweist es sich, dass der Architekt die Pläne jedes Mal vereinfacht hat, entweder aus eigener Initiative oder auf Anfrage des Auftraggebers. Der endgültige Bau Victor Blavettes entsprach einer im Umfang verringerten Version seines ursprünglichen Projektes. Der Architekt, der zwar auf den Zentralbau der Services Généraux verzichten musste, errichtete dennoch für die Fakultät der Wissenschaften ein Gebäude, das damals für seine besonders ehrgeizige und moderne Bauausführung weit gelobt wurde.

\section{AUTEUR}

\section{FLORENCE MARCIANO}

Florence Marciano, née en 1966, a obtenu un doctorat d'histoire de l'art et archéologie en 1999, sous la direction de Claude Massu, université de Provence sur la Formation et carrière des élèves de la classe d'architecture de l'École des beaux-arts à Marseille au XIX ${ }^{e}$ siècle. Chercheur associé au laboratoire de recherche histoire architecturale et urbaine - Sociétés (LADRHAUS), École nationale supérieure d'architecture de Versailles, ses recherches portent sur « L'architecture scolaire en région Ile-de-France. Étude des édifices scolaires, de la maternelle à la fin du 
secondaire construits à cet usage de 1830 à 1980 en région Ile-de-France. Deuxième phase :

Paris ", sous la responsabilité d'Anne-Marie Châtelet. En 2001, elle réalise 124 notices pour la base Mérimée, ministère de la culture : architecture du secteur Euroméditerranée à Marseille, puis des notices biographiques d'architectes marseillais pour la réédition de Les Marseillais dans l'histoire, Edisud : Gaudensi Allar, Marcel Dourgnon, Gabriel Héraud, Joseph Huot, Eugène Troump, Jean Séguéla. En 2005, publication de la troisième partie de la thèse de doctorat: Architecture domestique à Marseille au XIX ${ }^{e}$ siècle, du trois-fenêtres à l'Art Nouveau, Éd. La Thune, Marseille (avec la subvention du Conseil Général des Bouches-du-Rhône), qui obtient le prix Félix-de-Beaujour de l'Académie de Marseille, 2005. En 2006, elle publie : «Autour de la place Delibes », Revue Marseille, n ○214, octobre 2006. Adresse électronique : florence.marciano@wanadoo.fr 